\title{
Observer Based Output Feedback Control of Thrust Magnetic Bearings
}

\author{
Beytullah Okur, Erkan Zergeroglu, Enver Tatlicioglu, Sinan Basaran, and Selim Sivrioglu
}

\begin{abstract}
In this paper, we present an observer based output feedback controller for a thrust magnetic bearing system. A model independent variable structure like observer is used to determine the rotor velocity in order to remove the velocity dependency of the controller. The desired system dynamics have been utilized in the controller design and asymptotic stability of the observer-controller couple is guaranteed via Lyapunov based arguments. Experimental results are presented to illustrate the performance and feasibility of the proposed method.
\end{abstract}

\section{INTRODUCTION}

Active Magnetic Bearings (AMBs) consist of opposing electromagnets to support a rotor without physical contact. AMBs use magnetic forces to levitate a rotor and magnetic forces are naturally unstable [1]. Thus an AMB system have to be stabilized around an unstable equilibrium state of the overall system via a closed loop active controller. Magnetically levitated rotors have many useful advantages like noncontact and frictionless movement. Owing to these, AMBs allow rotors to reach high rotational speeds [2]. However due to the nature of magnetic field, magnetically levitated rotor systems are nonlinear and AMBs are represented by nonlinear mathematical expressions [3]. Nevertheless they can be linearized successfully around the operating point thanks to the restricted and very small air gap between AMB stator and rotor [1]. As a result many linear and local controllers like PID or fuzzy logic controllers [2], [4] have been successfully applied to AMB systems. These controllers can offer good performance around the operating point but outside these local regions where the effects of the nonlinearities become more evident, local controllers do not operate properly.

Model-based controllers are developed to increase the performance of the AMB system. But in model based controllers parametric uncertainties and measurement of the velocity become new issues to overcome. Parameters are very hard to define in magnetic bearings due to the magnetic saturation and eddy current effects. To our best knowledge nearly all AMBs do not have velocity sensors to measure rotor velocity

B. Okur is with the Department of Mechatronic Engineering, Yildiz Tech. University, Besiktas, Istanbul, Turkey.

beytullahokuregyte.edu.tr

E. Zergeroglu is with the Department of Computer Engineering, Gebze Institute of Technology, 41400, Gebze, Kocaeli, Turkey.

ezerger@bilmuh.gyte.edu.tr

E. Tatlicioglu is with the Department of Electrical \& Electronics Engineering, Izmir Institute of Technology, Gulbahce Koyu, Urla, Izmir, 35430 Turkey.

envertatliciogludiyte.edu.tr

S. Basaran and S. Sivrioglu are with the Department of Mechanical Engineering, Gebze Institute of Technology, 41400, Gebze, Kocaeli, Turkey. [sbarasan, s.sivrioglu] egyte.edu.tr in the AMB. The numerical derivation of the rotor position which is provided by very sensitive position sensors adds an evident noise to the closed loop control system.

In this study, we apply a model-based output feedback tracking controller with a model-free observer presented in [5] to the thrust magnetic bearing of a 5-axis flexible rotorAMB system. A continuous model-free observer formulation together with the desired system dynamics have been utilized in the controller design and global asymptotic stability of the observer-controller couple is guaranteed via Lyapunov based arguments.

The remaining of the paper is organized as follows: The mathematical model of a thrust magnetic bearing system is given in Section 2, while the problem formulation and error system development are stated in Section 3. Section 4 contains the observer-controller design with the stability analysis to ensure asymptotic tracking and boundedness of the closed-loop system. Results of the experimental studies performed on the thrust magnetic bearing of a 5 axis magnetic rotor levitation system are presented in Section 5. Concluding remarks are given in Section 6.

\section{Thrust Magnetic Bearing Model}

The linearized mathematical model for a one degree-offreedom thrust magnetic bearing (as shown in Figure 1) is assumed to have the following form [1]

$$
m \ddot{z}=k_{z} z+k_{i} i_{z}
$$

where $z(t), \ddot{z}(t) \in \mathbb{R}$ denote the rotor position and acceleration, respectively, $i_{z}(t) \in \mathbb{R}$ represents the control input current, $m \in \mathbb{R}$ represents the mass, $k_{i} \in \mathbb{R}$ is the current displacement factor, and $k_{z} \in \mathbb{R}$ is the force displacement factor.

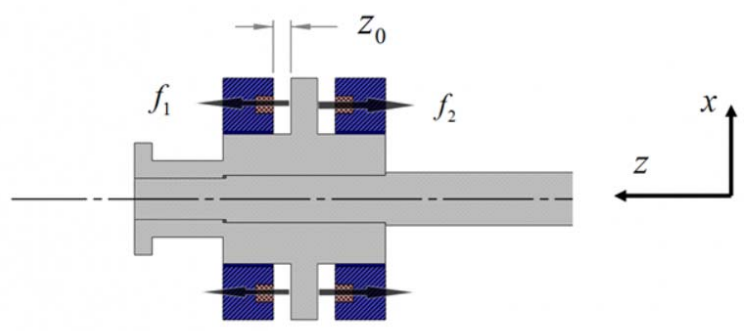

Fig. 1. Schematic Representation of a Magnetically Levitated Rotor

To ease the presentation of the subsequent control development, we divide both sides of (1) by the non-zero constant $k_{i}$ to obtain

$$
M \ddot{z}-C z=i_{z}
$$


where $M \triangleq m / k_{i}$ and $C \triangleq k_{z} / k_{i}$ are constant parameters. The modified rotor magnetic bearing system dynamics given in (2) can be written in terms of the desired rotor position and acceleration, denoted by $z_{d}(t), \ddot{z}_{d}(t) \in \mathbb{R}$, respectively, in the following manner

$$
W_{d} \triangleq M \ddot{z}_{d}-C z_{d}
$$

where $W_{d}\left(z_{d}, \ddot{z}_{d}\right) \in \mathbb{R}$ is assumed to be a known function.

\section{PROBLEM Formulation}

The control objective is to design an axial position tracking controller for horizontal rotor AMB systems under the restrictive constraint that only the rotor position variable $z(t)$ is available for control development.

We will quantify the control objective by defining the position tracking error, denoted by $e(t) \in \mathbb{R}$, as follows

$$
e \triangleq z_{d}-z \text {. }
$$

In the subsequent stability analysis, the standard assumption that $z_{d}(t)$ and its first three time derivatives being bounded functions of time will be utilized. To account for the unmeasurable velocity constraint, we define $\dot{\hat{z}}(t) \in \mathbb{R}$ as the observed velocity signal. The corresponding position and velocity observer error signals, denoted by $\tilde{z}(t), \dot{\tilde{z}}(t) \in \mathbb{R}$, respectively, are defined as

$$
\begin{aligned}
& \tilde{z} \triangleq z-\hat{z} \\
& \dot{\tilde{z}}=\dot{z}-\dot{\hat{z}} .
\end{aligned}
$$

To ease the presentation of the analysis, we will introduce filtered version of tracking error, denoted by $r(t) \in \mathbb{R}$, and filtered version of velocity observer error, denoted by $s(t) \in$ $\mathbb{R}$, as

$$
r \triangleq \dot{e}+\alpha e \text { and } s \triangleq \dot{\tilde{z}}+\alpha \tilde{z}
$$

where $\alpha \in \mathbb{R}$ is a positive control gain. It should be noted that, regulating $r(t)$ and $s(t)$ ensures the regulation of $e(t)$ and $\tilde{z}(t)$, respectively.

\section{ObServer-Controller Design}

Based on the subsequent error system development and the stability analysis, we propose the following velocity observer

$$
\begin{aligned}
\dot{\hat{z}} & =p+k_{0} \tilde{z}-k_{c} e \\
\dot{p} & =k_{1} \operatorname{sgn}(\tilde{z})+k_{2} \tilde{z}-\alpha k_{c} e
\end{aligned}
$$

where $p(t) \in \mathbb{R}$ is an auxiliary filter signal, $\operatorname{sgn}(\cdot) \in \mathbb{R}$ is the standard signum function, $k_{0}, k_{c}, k_{1}, k_{2} \in \mathbb{R}$ are positive gains and $\alpha$ was previously introduced in (7). It is straightforward to show that the time derivative of (8) yields

$$
\ddot{\tilde{z}}=k_{1} \operatorname{sgn}(\tilde{z})+k_{2} \tilde{z}+k_{0} \dot{\tilde{z}}-k_{c} r
$$

where the definition of $r(t)$ given in (7) was utilized. Based on the subsequent stability analysis, and after assuming exact knowledge of all the system parameters, the current input $i_{z}(t)$ is designed as

$$
i_{z}=W_{d}+k_{p} e+k_{c} \alpha\left(z_{d}-\hat{z}\right)+k_{c}\left(\dot{z}_{d}-\dot{\hat{z}}\right)
$$

where $k_{p} \in \mathbb{R}$ is a positive control gain. It should be noted that, after using the fact that

$$
z_{d}-\hat{z}=e+\tilde{z}
$$

the control input given in (11) can be rewritten in the following form

$$
i_{z}=W_{d}+k_{p} e+k_{c} r+k_{c} s
$$

which will be preferred in the subsequent stability analysis.

\section{A. Observer Analysis}

The observation error dynamics can be obtained as

$$
\begin{aligned}
\ddot{\tilde{z}} & =\ddot{z}-\ddot{\hat{z}} \\
& =N_{0}-k_{1} \operatorname{sgn}(\tilde{z})-k_{2} \tilde{z}-k_{0} \dot{\tilde{z}}+k_{c} r
\end{aligned}
$$

where (2) and (10) were utilized, and the auxiliary term $N_{0}(t) \in \mathbb{R}$ is defined as

$$
N_{0} \triangleq \frac{1}{M}\left(C z+i_{z}\right)
$$

After substituting (13) into (15), the following expression can be obtained

$$
N_{0}=N_{d}+N_{b}
$$

where (3) was utilized and the auxiliary variables $N_{d}(t)$, $N_{b}(t) \in \mathbb{R}$ are defined as

$$
\begin{aligned}
& N_{d} \triangleq \ddot{z}_{d} \\
& N_{b} \triangleq-\frac{C}{M} e+\frac{1}{M}\left(k_{p} e+k_{c} r+k_{c} s\right) .
\end{aligned}
$$

Remark 1: After exploiting the boundedness properties of the desired trajectory, we can show that $N_{d}(t)$ and its time derivative are bounded functions of time. Furthermore, the right-hand-side of (18) can be upper-bounded as follows

$$
\left|N_{b}(t)\right| \leq \rho_{01}|e|+\rho_{02}|r|+\rho_{03}|s|
$$

where $\rho_{0 i} \in \mathbb{R}, i=1,2,3$ are known positive bounding constants (see Appendix I).

After taking the time derivative of $s(t)$ in (7) and substituting (14), the dynamics for the filtered observation error $s(t)$ can be obtained as

$$
\dot{s}=N_{d}+N_{b}-k_{1} \operatorname{sgn}(\tilde{z})-k_{2} \tilde{z}-\left(k_{0}-\alpha\right) \dot{\tilde{z}}+k_{c} r .
$$

It should be noted that, when the observer gains are selected to satisfy

$$
\alpha\left(k_{0}-\alpha\right)=k_{2}
$$

the expression in (20) can be rearranged to have the following form

$$
\dot{s}=N_{d}+N_{b}-k_{1} \operatorname{sgn}(\tilde{z})-\frac{k_{2}}{\alpha} s+k_{c} r .
$$

The following preliminary Lyapunov-like analysis can be done for the observer design. Specifically, we define a non negative scalar function, denoted by $V_{0}(t) \in \mathbb{R}$, as follows

$$
V_{0}=\frac{1}{2} s^{2}+P_{0}
$$


where the scalar auxiliary function $P_{0}(t) \in \mathbb{R}$ is defined as

$$
P_{0} \triangleq \zeta_{0}-\int_{t_{0}}^{t} w_{0}(\sigma) d \sigma
$$

where $w_{0}(t) \in \mathbb{R}$ and the non-negative constant $\zeta_{0} \in \mathbb{R}$ are defined as

$$
\begin{aligned}
w_{0} & \triangleq s\left(N_{d}-k_{1} \operatorname{sgn}(\tilde{z})\right) \\
\zeta_{0} & \triangleq k_{1}\left|\tilde{z}\left(t_{0}\right)\right|-\tilde{z}\left(t_{0}\right) N_{d}\left(t_{0}\right) .
\end{aligned}
$$

Following a similar analysis ${ }^{1}$ to that of [6] and [7], it can be proven that when $k_{1}$ satisfies the following sufficient condition

$$
k_{1}>\left|N_{d}(t)\right|+\frac{1}{\alpha}\left|\dot{N}_{d}(t)\right|
$$

then $P_{0}(t)$ is always non-negative and $V_{0}(t)$ is a nonnegative Lyapunov function with respect to $s(t)$ and $\sqrt{P_{0}(t)}$. Taking the time derivative of (23) results in

$$
\dot{V}_{0}=s\left(-\frac{k_{2}}{\alpha} s+k_{c} r+N_{b}\right)
$$

where (22), time derivative of (24), and (25) were utilized. The first term in the brackets of (28) will be used for both damping the unwanted effects of the term $N_{b}(t)$ in the composite stability analysis and to ensure the convergence of the observation error. The second term is designed to cancel out the interconnection term between the observer/controller subsystem. At this point, we are ready to proceed to the error system development.

\section{B. Error System Development}

After taking the time derivative of $r(t)$, premultiplying the resulting equation with $M$, utilizing (2), (4), and performing some straightforward algebraic manipulation, the following expression can be obtained for the dynamics of $r(t)$

$$
M \dot{r}=W_{s}-i_{z}
$$

where the auxiliary term $W_{s}(t) \in \mathbb{R}$ is defined as

$$
W_{s} \triangleq M\left(\ddot{z}_{d}+\alpha \dot{e}\right)-C z .
$$

After substituting the control law (13) into (29), the following closed-loop dynamics for $r(t)$ can be obtained

$$
M \dot{r}=\chi-k_{c} r-k_{c} s-k_{p} e
$$

where the term $\chi(e, r, t) \in \mathbb{R}$ is defined as

$$
\chi \triangleq W_{s}-W_{d}
$$

where $W_{d}(\cdot)$ was previously defined in (3).

Remark 2: As illustrated in Appendix I, it can be shown that $\chi(\cdot)$ can be upper bounded as

$$
|\chi(\cdot)| \leq \rho_{1}|e|+\rho_{2}|r|
$$

where $\rho_{1}, \rho_{2} \in \mathbb{R}$ are known positive bounding constants. The above bound will be exploited in the stability analysis.

\footnotetext{
${ }^{1}$ Though the analysis very similar to that of the one given in the references, for the completeness of the presentation we have included it in Appendix II
}

\section{Stability Analysis}

The combination of error systems for (22) and (31) yields the following stability result for the observer error $\tilde{z}(t)$ and the position tracking error $e(t)$.

Theorem 1: The velocity observer in (9) and the control law in (11) ensure that the closed-loop observer/controller couple is globally asymptotically stable in the sense that

$$
|e(t)|,|\dot{\tilde{z}}(t)| \rightarrow 0 \text { as } t \rightarrow \infty
$$

provided that the controller and observer gains are selected to satisfy (21), (27) with controller gain $k_{c}$ and observer gain $k_{2}$ are chosen as

$$
\begin{aligned}
& k_{c}=1+\rho_{2}+k_{n} \rho_{1}^{2} \\
& k_{2}=\alpha\left(1+\rho_{o 3}+k_{n}\left(\rho_{o 1}^{2}+\rho_{o 2}^{2}\right)\right)
\end{aligned}
$$

where $k_{n} \in \mathbb{R}$ is a nonlinear damping gain, $\rho_{1}, \rho_{2}$ and $\rho_{o i}$, $i=1,2,3$ were introduced in (33) and (19), respectively.

Proof: We start our proof by introducing a non-negative function, denoted by $V(t) \in \mathbb{R}$, as follows

$$
V=V_{0}+\frac{1}{2} M r^{2}+\frac{1}{2} k_{p} e^{2} .
$$

From (37), $V(t)$ can be upper and lower bounded as

$$
\lambda_{1}\|x\|^{2} \leq \lambda_{1}\|y\|^{2} \leq V(y) \leq \lambda_{2}\|y\|^{2}
$$

where $x(t) \in \mathbb{R}^{3}$ and $y(t) \in \mathbb{R}^{4}$ are defined as follows

$$
\begin{aligned}
& x \triangleq\left[\begin{array}{lll}
s & r & e
\end{array}\right]^{T} \\
& y \triangleq\left[\begin{array}{llll}
s & \sqrt{P_{0}} & r & e
\end{array}\right]^{T}
\end{aligned}
$$

and the positive constants $\lambda_{1}, \lambda_{2} \in \mathbb{R}$ are defined as

$$
\begin{aligned}
& \lambda_{1} \triangleq \frac{1}{2} \min \left\{1, M, k_{p}\right\} \\
& \lambda_{2} \triangleq \frac{1}{2} \max \left\{2, M, k_{p}\right\} .
\end{aligned}
$$

After taking the time derivative of (37), we obtain

$$
\dot{V}=s\left(-\frac{k_{2}}{\alpha} s+N_{b}\right)+r\left(\chi-k_{c} r\right)-\alpha k_{p} e^{2}
$$

where (7), (28) and (31) were utilized. After substituting (19), (33), (35) and (36) into (43), the following upper bound can be formed

$$
\begin{aligned}
\dot{V} \leq & -k_{p} \alpha|e|^{2}-|r|^{2}-|s|^{2} \\
& +\left(\rho_{01}|e||s|-k_{n} \rho_{01}^{2}|s|^{2}\right) \\
& +\left(\rho_{02}|r||s|-k_{n} \rho_{02}^{2}|s|^{2}\right) \\
& +\left(\rho_{1}|r||e|-k_{n} \rho_{1}^{2}|r|^{2}\right) .
\end{aligned}
$$

After completing the squares of the terms in the brackets we obtain

$$
\dot{V} \leq-\left(k_{p} \alpha-\frac{1}{2 k_{n}}\right)|e|^{2}-\left(1-\frac{1}{4 k_{n}}\right)|r|^{2}-|s|^{2}
$$

which, after using the definition of $x(t)$ in (39), can be further upper bounded as

$$
\dot{V} \leq-\beta\|x\|^{2}
$$


where $\beta \in \mathbb{R}$ is some positive constant.

That is, when the gain conditions in (21), (27), (35) and (36) are satisfied, $V(t) \in \mathcal{L}_{\infty}$; thus, $y(t), x(t)$, $e(t), r(t), s(t) \in \mathcal{L}_{\infty}$. Following standard signal chasing arguments, it can be shown that all closed-loop signals are bounded, and $e(t)$ and $\dot{\tilde{z}}(t)$ are uniformly continuos (from the boundedness of their derivatives over time). Furthermore, from the integration of both sides of (46), it is easy to see that $x(t) \in \mathcal{L}_{2}$ and therefore $e(t), \dot{\tilde{z}}(t) \in \mathcal{L}_{2}$. Finally, after utilizing a direct application of Barbalat's Lemma [8], we can obtain the result given in (34) provided that the gain conditions are satisfied.

\section{EXPERIMENTAL RESULTS}

To illustrate the performance of the proposed control scheme, we have implemented the proposed observercontroller couple on the thrust magnetic bearing of a 5 degree of freedom magnetic bearing system (shown in Figure 2) manufactured by the The Shanghai Bo-hong Science and Manufacturing Trade Corporation. The overall system is composed of 3 magnetic bearing (one thrust and two radial) and is driven by 5 individual amplifiers. Capacitive sensors are used to measure the position of each magnetic bearing. The experiments were performed on the thrust magnetic bearing while the radial magnetic bearings were controlled by conventional PID controllers. The controller algorithm were implemented using Matlab/Simulink simulation environment while the data acquisition and controller implementation were performed using dSpace ACE Kit 1103 system. The observer and controller gains were selected as

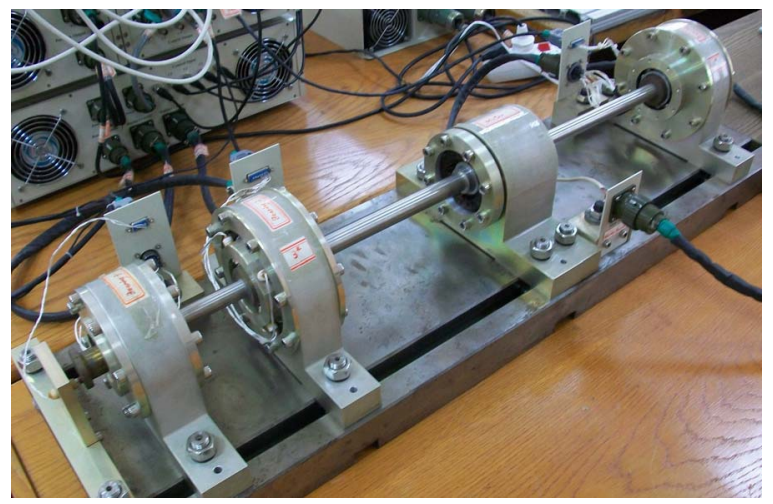

Fig. 2. 5 Degree of Freedom Magnetic Bearing Experimental Setup

$$
\begin{aligned}
\alpha & =2 \\
k_{0} & =1200, k_{c}=150 \\
k_{1} & =2, \text { and } k_{p}=200000 .
\end{aligned}
$$

The desired end position of the rotor is selected as the midpoint of the bearing (i.e. $z_{d}=0$ ). The position observation error during the experiments are presented in Figure 3. Figure 4 shows the position tracking error while the control input to the system is presented in Figure 5. As can be seen form the Figures the data logging starts at time $t=0$ while the controller action starts around $t=0.4 \mathrm{sec}$ and regulation of the system around the desired end point is obtained very quickly.

\section{CONCLUSION}

In this work, we have presented an observer based controller formulation for the output feedback control of thrust magnetic bearing systems. Specifically a model independent variable structure like observer in conjunction with the desired system dynamics have been utilized to remove the velocity dependency of the controller. Despite the lack of velocity measurement the proposed observer-controller couple achieves global asymptotic stability. Stability of the proposed method have been proven via Lyapunov based arguments and experimental results are presented to illustrate the performance and feasibility of the proposed method.

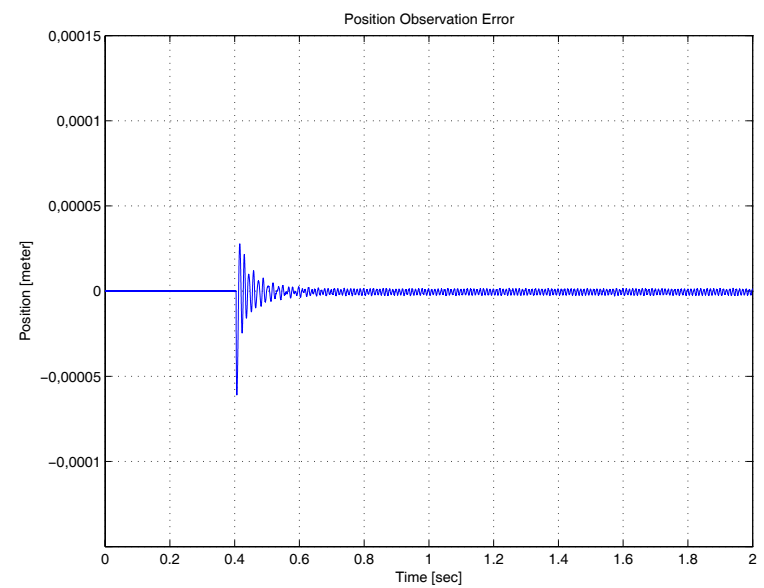

Fig. 3. The position observation error

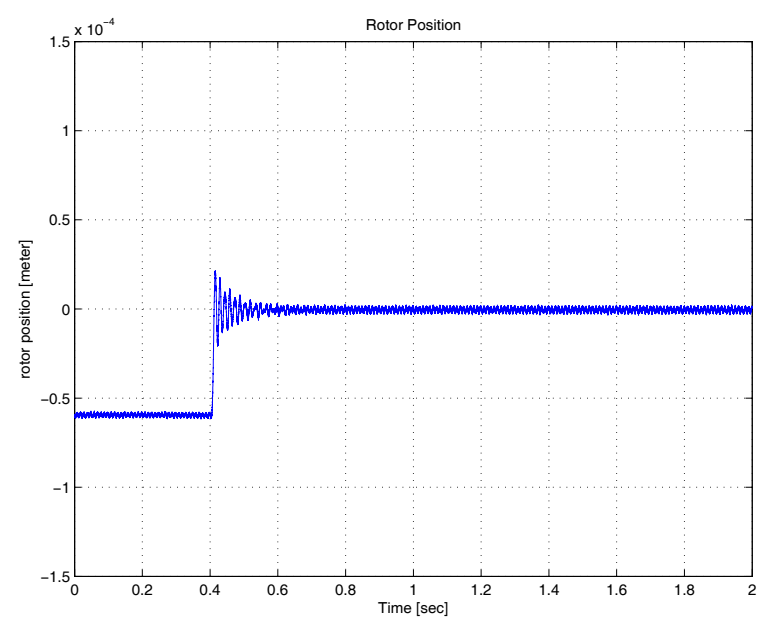

Fig. 4. The positioning error signal

\section{REFERENCES}

[1] A. Chiba, T. Fukao, O. Ichikawa, M. Osahima, M. Takemoto, and D. Dorrell, Magnetic Bearings and Bearingless Drives. Tokyo, Japan: Elsevier, 2005. 


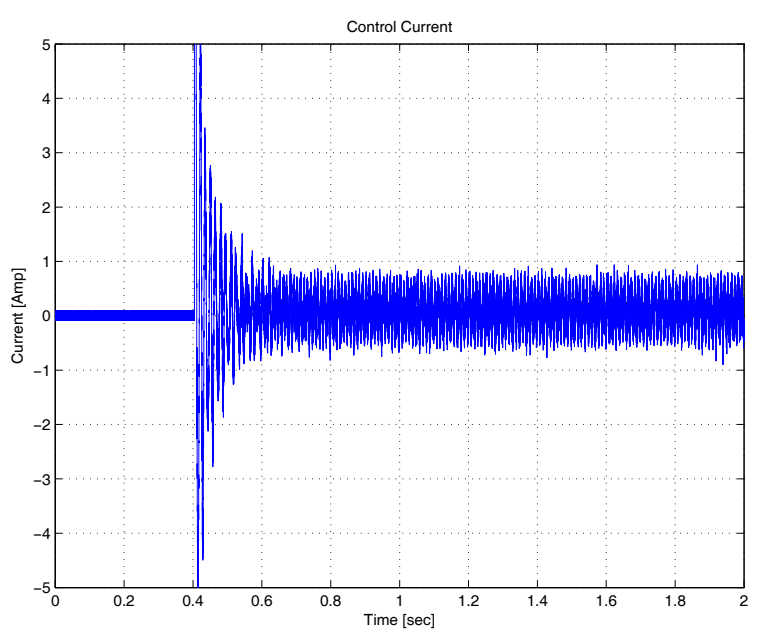

Fig. 5. The controller current input to the system

[2] M. Murata, H. Tijama, T. Watanabe, and K. Seto, "New modeling and control methods for flexible rotors with magnetic bearings toward passing through critical speeds caused by elastic modes," in Int. Symp. on Magnetic Bearings, Martigny, Switzerland, 2006.

[3] Z. Yu, D. Wen, and H. Zhang, "The identification model of magnetic bearing supporting system," in IEEE Int. Conf. on Computer Science \& Software Engineering, Wuhan, China, 2008, pp. 70-73.

[4] T. P. Minihan, S. Lei, G. Sun, A. Palazzolo, A. F. Kascak, and T. Calvert, "Large motion tracking control for thrust magnetic bearings with fuzzy logic, sliding mode, and direct linearizaton," J. of Sound \& Vibration, vol. 263, no. 3, pp. 549-567, 2003.

[5] E. Zergeroglu and E. Tatlicioglu, "Observer based output feedback tracking control of robot manipulators," in Proc. of IEEE Int. Conf. on Control Applications, Yokohama, Japan, 2010, pp. 602-607.

[6] B. Xian, D. M. Dawson, M. S. de Queiroz, and J. Chen, "A continuous asymptotic tracking control strategy for uncertain nonlinear systems," IEEE Trans. Automat. Contr., vol. 49, no. 7, pp. 1206-1211, 2004.

[7] B. Xian, M. S. de Queiroz, D. M. Dawson, and M. L. McIntyre, "Output feedback variable structure-like control of nonlinear mechanical systems," in Proc. IEEE Int. Conf. Decision and Control, Maui, HA, USA, 2003, pp. 368-373.

[8] M. Krstic, I. Kanellakopoulos, and P. Kokotovic, Nonlinear and Adaptive Control Design. New York, NY, USA: John Wiley and Sons, 1995.

\section{APPENDIX I}

\section{PROOF OF BOUNDS}

In this appendix, we illustrate how the upper bounds of $N_{b}(t)$ in (19) and $\chi(t)$ in (33) are obtained.

We start with exploting the expression given in (18), which can be rewritten in the following form

$$
N_{b}=\frac{k_{p}-C}{M} e+\frac{k_{c}}{M} r+\frac{k_{c}}{M} s .
$$

From the structure of (47), it is clear that the bounding constants $\rho_{o i}, i=1,2,3$ in (19) are defined as

$$
\rho_{01} \triangleq\left|\frac{k_{p}-C}{M}\right|, \rho_{02} \triangleq \frac{k_{c}}{M}, \rho_{03} \triangleq \frac{k_{c}}{M} .
$$

To obtain the upper bound in (33), we start with substituting (3) and (30) into (32) to obtain the following expression

$$
\chi=\left(C-\alpha^{2} M\right) e+\alpha M r
$$

where the definitions of $e(t)$ and $r(t)$ in (4) and (7), respectively, were utilized. From the structure of (49), it is clear that the bounding constants $\rho_{1}$ and $\rho_{2}$ in (33) are defined as

$$
\rho_{1} \triangleq\left|C-\alpha^{2} M\right| \text { and } \rho_{2} \triangleq \alpha M
$$

\section{APPENDIX II}

\section{THE GAIN CONDITION OF $k_{1}$}

In this appendix, we will illustrate how the sufficient condition of (27) is obtained. After substituting the definition of $s(t)$ in (7) into (25) and then integrating $w_{0}(t)$ in time results in the following expression

$$
\begin{aligned}
\int_{t_{0}}^{t} w_{0}(\sigma) d \sigma= & \int_{t_{0}}^{t} \tilde{z}(\sigma) \alpha\left(N_{d}(\sigma)-k_{1} \operatorname{sgn}(\tilde{z}(\sigma))\right) d \sigma \\
& +\int_{t_{0}}^{t} \frac{d \tilde{z}(\sigma)}{d \sigma} N_{d}(\sigma) d \sigma \\
& -k_{1} \int_{t_{0}}^{t} \frac{d \tilde{z}(\sigma)}{d \sigma} \operatorname{sgn}(\tilde{z}(\sigma)) d \sigma
\end{aligned}
$$

After integrating the second integral on the right-hand-side of (51) by parts, the following expression is obtained

$$
\begin{aligned}
\int_{t_{0}}^{t} w_{0}(\sigma) d \sigma & =\int_{t_{0}}^{t} \tilde{z}(\sigma) \alpha\left(N_{d}(\sigma)-k_{1} \operatorname{sgn}(\tilde{z}(\sigma))\right) d \sigma \\
& +\left.\left(\tilde{z}(\sigma) N_{d}(\sigma)\right)\right|_{t_{0}} ^{t}-\int_{t_{0}}^{t} \tilde{z}(\sigma) \frac{d N_{d}(\sigma)}{d \sigma} d \sigma \\
& -\left.k_{1}(|\tilde{z}(\sigma)|)\right|_{t_{0}} ^{t} .
\end{aligned}
$$

After combining common terms, from (52), the following expression can be obtained

$$
\begin{aligned}
\int_{t_{0}}^{t} w_{0}(\sigma) d \sigma= & \int_{t_{0}}^{t} \tilde{z}(\sigma) \alpha\left[N_{d}(\sigma)\right. \\
& \left.-\frac{1}{\alpha} \frac{d N_{d}(\sigma)}{d \sigma}-k_{1} \operatorname{sgn}(\tilde{z}(\sigma))\right] d \sigma \\
& +\tilde{z}(t) N_{d}(t)-\tilde{z}\left(t_{0}\right) N_{d}\left(t_{0}\right) \\
& -k_{1}\left(|\tilde{z}(t)|-\left|\tilde{z}\left(t_{0}\right)\right|\right) .
\end{aligned}
$$

The right-hand-side of (53) can be upper bounded as follows

$$
\begin{aligned}
\int_{t_{0}}^{t} w_{0}(\sigma) d \sigma \leq & \int_{t_{0}}^{t}|\tilde{z}(\sigma)| \alpha\left[\left|N_{d}(\sigma)\right|\right. \\
& \left.+\frac{1}{\alpha}\left|\frac{d N_{d}(\sigma)}{d \sigma}\right|-k_{1}\right] d \sigma \\
& +|\tilde{z}(t)|\left(\left|N_{d}(t)\right|-k_{1}\right)+\zeta_{0} .
\end{aligned}
$$

If $k_{1}$ is chosen to satisfy (27), from (54), it is easy to obtain the following expression

$$
\int_{t_{0}}^{t} w_{0}(\sigma) d \sigma \leq \zeta_{0}
$$

thus; from (24), it can be concluded that $P_{0}(t)$ is nonnegative. 